\title{
Long-Distance Quantum Communication
}

\author{
L. Duan ${ }^{a}$, M. Lukin ${ }^{b}$, J.I. Cirac ${ }^{a}$ And P. Zoller ${ }^{b}$ \\ ${ }^{a}$ Institute for Theoretical Physics, University of Innsbruck \\ Technikerstrasse 25-2, 6020 Innsbruck, Austria \\ ${ }^{b}$ Physics Department, University of Harvard, Cambridge, MA, USA \\ We show how one can use atomic ensembles in order to construct quan- \\ tum repeaters. This may allow for quantum communication over arbitrarily \\ long distances.
}

PACS numbers: $03.67 .-\mathrm{a}, 42.50 .-\mathrm{p}$

\section{Introduction}

Quantum communication is an essential element required for constructing quantum networks, and it also has the application for absolutely secret transfer of classical messages by means of quantum cryptography [1]. The central problem of quantum communication is to generate nearly perfect entangled states between distant sites. Such states can be used, for example, to implement secure quantum cryptography using the Ekert protocol [1], and to faithfully transfer quantum states via quantum teleportation [2]. All the known realistic schemes for quantum communication are based on the use of the photonic channels. However, the degree of entanglement generated between two distant sites normally decreases exponentially with the length of the connecting channel due to the optical absorption and other channel noise. To regain a high degree of entanglement, purification schemes can be used [3]. However, entanglement purification does not fully solve the long-distance quantum communication problem. Due to the exponential decay of the entanglement in the channel, one needs an exponentially large number of partially entangled states to obtain one highly entangled state, which means that for a sufficiently long distance the task becomes nearly impossible.

To overcome the difficulty associated with the exponential fidelity decay, the concept of quantum repeaters can be used [4]. In principle, it allows to make the overall communication fidelity very close to the unity, with the communication time growing only polynomially with the transmission distance. In analogy to a 
fault-tolerant quantum computing $[5,6]$, the quantum repeater proposal is a cascaded entanglement purification protocol for communication systems. The basic idea is to divide the transmission channel into many segments, with the length of each segment comparable to the channel attenuation length. First, one generates entanglement and purifies it for each segment; the purified entanglement is then extended to a longer length by connecting two adjacent segments through entanglement swapping [2, 7]. After entanglement swapping, the overall entanglement is decreased, and one has to purify it again. One can continue the rounds of the entanglement swapping and purification until nearly perfect entangled states are created between two distant sites.

To implement the quantum repeater protocol, one needs to generate entanglement between distant quantum bits (qubits), store them for sufficiently long time and perform local collective operations on several of these qubits. The requirement of quantum memory is essential since all purification protocols are probabilistic. When entanglement purification is performed for each segment of the channel, quantum memory can be used to keep the segment state if the purification succeeds and to repeat the purification for the segments only where the previous attempt fails. This is essentially important for polynomial scaling properties of the communication efficiency since with no available memory we have to require that the purifications for all the segments succeed at the same time; the probability of such event decreases exponentially with the channel length. The requirement of quantum memory implies that we need to store the local qubits in the atomic internal states instead of the photonic states since it is difficult to store photons for a reasonably long time. With atoms as the local information carriers it seems to be very hard to implement quantum repeaters since normally one needs to achieve the strong coupling between atoms and photons with high-finesse cavities for atomic entanglement generation, purification, and swapping [8, 9], which, in spite of the recent significant experimental advances $[10,12]$, remains a very challenging technology.

To overcome this difficulty, Ref. [13] proposes a very different scheme to realize quantum repeaters based on the use of atomic ensembles with the $\Lambda$ II-level configuration. The laser manipulation of the atomic ensembles, together with some simple linear optics devices and moderate single-photon detectors, do the whole work for long-distance quantum communication. The setup is much simpler compared with the single-atom and high- $Q$ cavity approach discussed above. To achieve this, the scheme makes significant advances in each step of entanglement generation, connection, and applications, with each step having built-in entanglement purification and resilient to the realistic noise. As a result, the scheme circumvents the realistic noise and imperfections, and at the same time keeps the overhead in the communication time increasing with the distance only polynomially. In this paper, we will review the realization of quantum repeaters and long-distance quantum communication following the approach in Ref. [13]. 


\section{Entanglement generation}

To realize long-distance quantum communication, first we need to entangle two atomic ensembles within the channel attenuation length. The entanglement generation scheme described here is based on single-photon interference at photodetectors, and is fault-tolerant to realistic noise. This scheme is an extension of a proposal first proposed in $[14,15]$ to entangle single atoms. The extension was made in [13] to entangle atomic ensembles with significant improvements in the communication efficiency thanks to the collective enhancement of the signal-to-noise ratio for many-atom ensembles.

The system is a sample of atoms prepared in the ground state $|1\rangle$ with the AII-level configuration (see Fig. 1). It can be shown that one can define an effective single-mode bosonic annihilation operator $a$ for the cavity output signal (it is called the forward-scattered Stokes signal in the free space case). After the light-atom interaction, the signal mode $a$ and the collective atomic mode

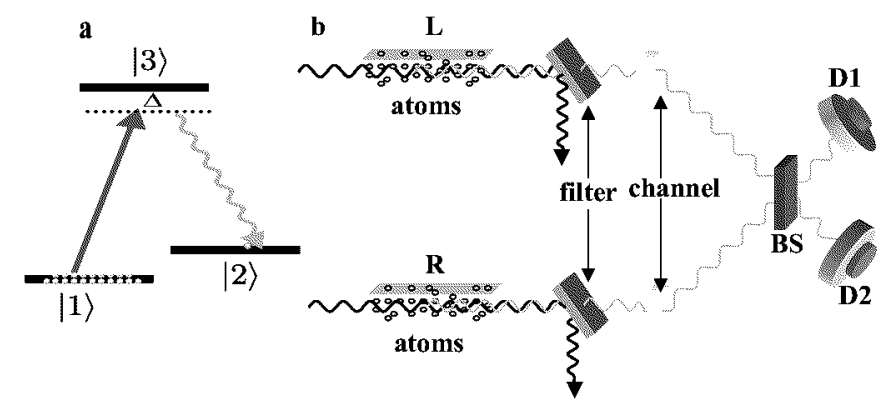

Fig. 1. (a) The relevant level structure of the atoms in the ensemble with $|1\rangle$, the ground state, $|2\rangle$, the metastable state for storing a qubit, and $|3\rangle$, the excited state. The transition $|1\rangle \rightarrow|3\rangle$ is coupled by the classical laser with the Rabi frequency $\Omega$, and the forward scattering Stokes light comes from the transition $|3\rangle \rightarrow|2\rangle$. For convenience, we assume off-resonant coupling with a large detuning $\Delta$. (b) Schematic setup for generating entanglement between the two atomic ensembles $L$ and $R$. The two ensembles are pencil shaped and illuminated by the synchronized classical laser pulses. The forward-scattering Stokes pulses are collected after the filters (polarization and frequency selective) and interfered at a 50\%-50\% beam splitter $B S$ after the transmission channels, with the outputs detected respectively by two single-photon detectors $D 1$ and $D 2$. If there is a click in $D 1$ or $D 2$, the process is finished and we successfully generate entanglement between the ensembles $L$ and $R$. Otherwise, we first apply a repumping pulse to the transition $|2\rangle \rightarrow|3\rangle$ on the ensembles $L$ and $R$ to set the state of the ensembles back to the ground state $|0\rangle_{a}^{L} \otimes|0\rangle_{a}^{R}$, then the same classical laser pulses as the first round are applied to the transition $|1\rangle \rightarrow|3\rangle$ and we detect again the forward-scattering Stokes pulses after the beam splitter. This process is repeated until finally we have a click in the $D 1$ or $D 2$ detector. 
$s \equiv\left(1 / \sqrt{N_{a}}\right) \sum_{i}|1\rangle_{i}\langle 2|$ are in a two-mode squeezed state with the squeezing parameter $r_{c}$ proportional to the interaction time $t_{\Delta}$. If the interaction time $t_{\Delta}$ is very small, the whole state of the collective atomic mode and the signal mode can be written in the perturbative form

$$
|\phi\rangle=\left|0_{a}\right\rangle\left|0_{p}\right\rangle+\sqrt{p_{c}} S^{\dagger} a^{\dagger}\left|0_{a}\right\rangle\left|0_{p}\right\rangle+\mathrm{O}\left(p_{c}\right),
$$

where $p_{c}=\tanh ^{2} r_{c}$ is the small excitation probability and $\mathrm{O}\left(p_{c}\right)$ represents the terms with more excitations whose probabilities are equal or smaller than $p_{c}^{2}$. The $\left|0_{a}\right\rangle$ and $\left|0_{p}\right\rangle$ are respectively the atomic and optical vacuum states with $\left|0_{a}\right\rangle \equiv \bigotimes_{i}|1\rangle_{i}$. There is also a fraction of light from the transition $|3\rangle \rightarrow|2\rangle$ emitted in other directions which contributes to spontaneous emissions. It can be shown that the contribution to the population in the collective atomic mode $s$ from the spontaneous emissions is very small for many-atom ensembles due to the collective enhancement of the signal-to-noise ratio for this mode.

Now we show how to use this setup to generate entanglement between two distant ensembles $L$ and $R$ using the configuration shown in Fig. 1. Here, two laser pulses excited both ensembles simultaneously, and the whole system is described by the state $|\phi\rangle_{L} \otimes|\phi\rangle_{R}$, where $|\phi\rangle_{L}$ and $|\phi\rangle_{R}$ are given by Eq. (1) with all the operators and states distinguished by the subscript $L$ or $R$. The forward scattered Stokes signal from both ensembles is combined at the beam splitter and a photodetector click in either $D 1$ or $D 2$ measures the combined radiation from two samples, $a_{+}^{\dagger} a_{+}$ or $a_{-}^{\dagger} a_{-}$with $a_{ \pm}=\left(a_{L} \pm \mathrm{e}^{\mathrm{i} \varphi} a_{R}\right) / \sqrt{2}$. Here, $\varphi$ denotes an unknown difference of the phase shifts in the two-side channels. We can also assume that $\varphi$ has an imaginary part to account for the possible asymmetry of the setup, which will also be corrected automatically in our scheme. But the setup asymmetry can be easily made very small, and for simplicity of expressions we assume that $\varphi$ is real in the following. Conditional on the detector click, we should apply $a_{+}$or $a_{-}$to the whole state $|\phi\rangle_{L} \otimes|\phi\rangle_{R}$, and the projected state of the ensembles $L$ and $R$ is nearly maximally entangled with the form (neglecting the high-order terms $\mathrm{O}\left(p_{c}\right)$ )

$$
\left|\Psi_{\varphi}\right\rangle_{L R}^{ \pm}=\left[\left(S_{L}^{\dagger} \pm \mathrm{e}^{\mathrm{i} \varphi} S_{R}^{\dagger}\right) / \sqrt{2}\right]\left|0_{a}\right\rangle_{L}\left|0_{a}\right\rangle_{R}
$$

The probability for getting a click is given by $p_{c}$ for each round, so we need to repeat the process about $1 / p_{c}$ times for a successful entanglement preparation, and the average preparation time is given by $T_{0} \sim t_{\Delta} / p_{c}$. The states $\left|\Psi_{r}\right\rangle_{L R}^{+}$and $\left|\Psi_{r}\right\rangle_{L R}^{-}$ can be easily transformed to each other by a simple local phase shift. Without loss of generality, we assume in the following that we generate the entangled state $\left|\psi_{r}\right\rangle_{L R}^{+}$

As will be shown below, the presence of the noise modifies the projected state of the ensembles to

$$
\rho_{L R}\left(c_{0}, \varphi\right)=\frac{1}{c_{0}+1}\left(c_{0}\left|0_{a} 0_{a}\right\rangle_{L R}\left\langle 0_{a} 0_{a}|+| \Psi_{\varphi}\right\rangle_{L R}^{+}\left\langle\Psi_{\varphi}\right|\right),
$$

where the "vacuum" coefficient $c_{0}$ is determined by the dark count rates of the photon detectors. It will be seen below that any state in the form of Eq. (3) will be 
purified automatically to a maximally entangled state in the entanglement-based communication schemes. We therefore call this state an effective maximally entangled (EME) state with the vacuum coefficient $c_{0}$ determining the purification efficiency.

\section{Entanglement connection through swapping}

After the successful generation of the entanglement within the attenuation length, we want to extend the quantum communication distance. This is done through entanglement swapping with the configuration shown in Fig. 2. Let us suppose that we start with two pairs of the entangled ensembles described by the state $\rho_{L I_{1}} \otimes \rho_{I_{2} R}$, where $\rho_{L I_{1}}$ and $\rho_{I_{2} R}$ are given by Eq. (3). In the ideal case, the setup shown in Fig. 2 measures the quantities corresponding to operators $S_{ \pm}^{\dagger} S_{ \pm}$with $S_{ \pm}=\left(S_{I_{1}} \pm S_{I_{2}}\right) / \sqrt{2}$. If the measurement is successful (i.e., one of the detectors registers one photon), we will prepare the ensembles $L$ and $R$ into another EME state. The new $\varphi$-parameter is given by $\varphi_{1}+\varphi_{2}$, where $\varphi_{1}$ and $\varphi_{2}$ denote the old $\varphi$-parameters for the two segment EME states. As will be seen below, even in the presence of the realistic noise and imperfections, an EME state is

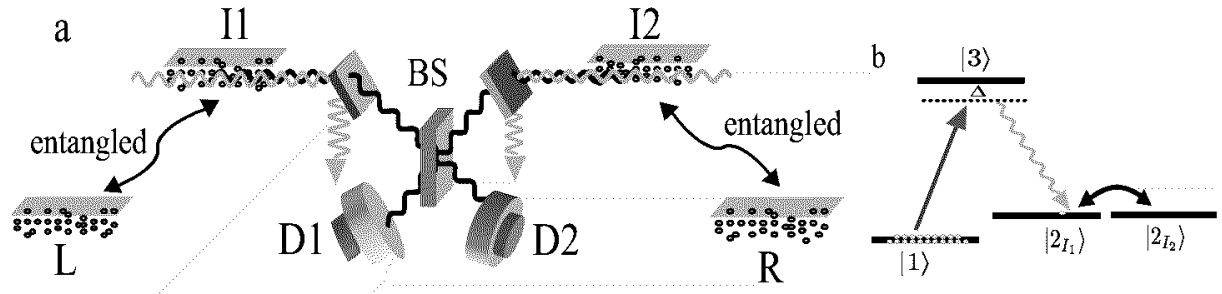

Fig. 2. (a) Illustrative setup for the entanglement swapping. We have two pairs of ensembles $L, I_{1}$ and $I_{2}, R$ distributed at three sites $L, I$ and $R$. Each of the ensemble-pairs $L, I_{1}$ and $I_{2}, R$ is prepared in an EME state in the form of Eq. (3). The excitations in the collective modes of the ensembles $I_{1}$ and $I_{2}$ are transferred simultaneously to the optical excitations by the repumping pulses applied to the atomic transition $|2\rangle \rightarrow|3\rangle$, and the stimulated optical excitations, after a 50\%-50\% beam splitter, are detected by the single-photon detectors $D 1$ and $D 2$. If either $D 1$ or $D 2$ clicks, the protocol is successful and an EME state in the form of Eq. (3) is established between the ensembles $L$ and $R$ with a doubled communication distance. Otherwise, the process fails, and we need to repeat the previous entanglement generation and swapping until finally we have a click in $D 1$ or $D 2$, that is, until the protocol finally succeeds. (b) The two intermediated ensembles $I_{1}$ and $I_{2}$ can also be replaced by one ensemble but with two metastable states $I_{1}$ and $I_{2}$ to store the two different collective modes. The $50 \%-50 \%$ beam splitter operation can be simply realized by a $\pi / 2$ pulse on the two metastable states before the collective atomic excitations are transferred to the optical excitations. 
still created after a detector click. The noise only influences the success probability to get a click and the new vacuum coefficient in the EME state. In general, we can express the success probability $p_{1}$ and the new vacuum coefficient $c_{1}$ as $p_{1}=f_{1}\left(c_{0}\right)$ and $c_{1}=f_{2}\left(c_{0}\right)$, where the functions $f_{1}$ and $f_{2}$ depend on the particular noise properties.

The above method for connecting entanglement can be cascaded to arbitrarily extend the communication distance. For the $i$-th $(i=1,2, \ldots, n)$ entanglement connection, we first prepare in parallel two pairs of ensembles in the EME states with the same vacuum coefficient $c_{i-1}$ and the same communication length $L_{i-1}$, and then perform the entanglement swapping as shown in Fig. 2, which now succeeds with a probability $p_{i}=f_{1}\left(c_{i-1}\right)$. After a successful detector click, the communication length is extended to $L_{i}=2 L_{i-1}$, and the vacuum coefficient in the connected EME state becomes $c_{i}=f_{2}\left(c_{i-1}\right)$. Since the $i$-th entanglement connection needs to be repeated in average $1 / p_{i}$ times, the total time needed to establish an EME state over the distance $L_{n}=2^{n} L_{0}$ is given by $T_{n}=T_{0} \prod_{i=1}^{n}\left(1 / p_{i}\right)$, where $L_{0}$ denotes the distance of each segment in the entanglement generation.

\section{Entanglement-based communication schemes}

After an EME state has been established between two distant sites, we would like to use it in the communication protocols, such as quantum teleportation, cryptography, and the Bell inequality detection. It is not obvious that the EME state (3), which is entangled in the Fock basis, is useful for these tasks since in the Fock basis it is experimentally hard to do certain single-bit operations. In the following we will show how the EME states can be used to realize all these protocols with simple experimental configurations.

Quantum cryptography and the Bell inequality detection are achieved with the setup shown by Fig. 3a. The state of the two pairs of ensembles is expressed as $\rho_{L_{1} R_{1}} \otimes \rho_{L_{2} R_{2}}$, where $\rho_{L_{i} R_{i}}(i=1,2)$ denote the same EME state with the vacuum coefficient $c_{n}$ if we have done $n$ times entanglement connection. The $\varphi$-parameters in $\rho_{L_{1} R_{1}}$ and $\rho_{L_{2} R_{2}}$ are the same provided that the two states are established over the same stationary channels. We register only the coincidences of the two-side detectors, so the protocol is successful only if there is a click on each side. Under this condition, the vacuum components in the EME states, together with the state components $S_{L_{1}}^{\dagger} S_{L_{2}}^{\dagger}|\mathrm{vac}\rangle$ and $S_{R_{1}}^{\dagger} S_{R_{2}}^{\dagger}|\mathrm{vac}\rangle$, where $|\mathrm{vac}\rangle$ denotes the ensemble state $\left|0_{a} 0_{a} 0_{a} 0_{a}\right\rangle_{L_{1} R_{1} L_{2} R_{2}}$, have no contributions to the experimental results. Therefore, for the measurement scheme shown by Fig. 3 , the ensemble state $\rho_{L_{1} R_{1}} \otimes \rho_{L_{2} R_{2}}$ is effectively equivalent to the following "polarization" maximally entangled (PME) state (the terminology of "polarization" comes from an analogy to the optical case)

$$
|\Psi\rangle_{\mathrm{PME}}=\left[\left(S_{L_{1}}^{\dagger} S_{R_{2}}^{\dagger}+S_{L_{2}}^{\dagger} S_{R_{1}}^{\dagger}\right) / \sqrt{2}\right]|\mathrm{vac}\rangle
$$

The success probability for the projection from $\rho_{L_{1} R_{1}} \otimes \rho_{L_{2} R_{2}}$ to $|\Psi\rangle_{\mathrm{PME}}$ (i.e., the probability to get a click on each side) is given by $p_{a}=1 /\left[2\left(c_{n}+1\right)^{2}\right]$. One 


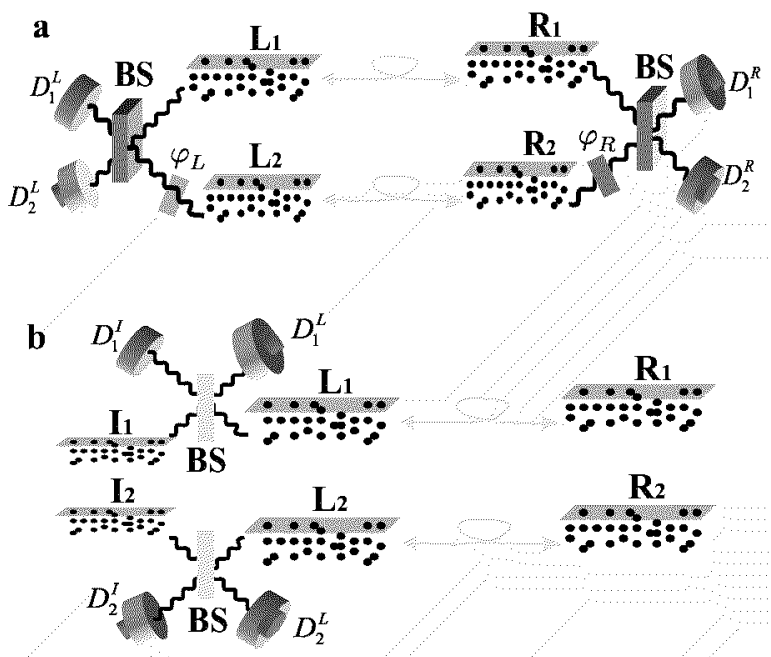

Fig. 3. (a) Schematic setup for the realization of quantum cryptography and Bell inequality detection. Two pairs of ensembles $L_{1}, R_{1}$ and $L_{2}, R_{2}$ (or two pairs of metastable states as shown in Fig. 1b) have been prepared in the EME states. The collective atomic excitations on each side are transferred to the optical excitations, which, respectively after a relative phase shift $\varphi_{L}$ or $\varphi_{R}$ and a $50 \%-50 \%$ beam splitter, are detected by the single-photon detectors $D_{1}^{L}, D_{2}^{L}$ and $D_{1}^{R}, D_{2}^{R}$. We look at the four possible coincidences of $D_{1}^{R}, D_{2}^{R}$ with $D_{1}^{L}, D_{2}^{L}$, which are functions of the phase difference $\varphi_{L}-\varphi_{R}$. Depending on the choice of $\varphi_{L}$ and $\varphi_{R}$, this setup can realize both the quantum cryptography and the Bell inequality detection. (b) Schematic setup for probabilistic quantum teleportation of the atomic "polarization" state. Similarly, two pairs of ensembles $L_{1}, R_{1}$ and $L_{2}, R_{2}$ are prepared in the EME states. We want to teleport an atomic "polarization" state $\left(d_{0} S_{I_{1}}^{\dagger}+d_{1} S_{I_{2}}^{\dagger}\right)\left|0_{a} 0_{a}\right\rangle_{I_{1} I_{2}}$ with unknown coefficients $d_{0}, d_{1}$ from the left to the right side, where $S_{I_{1}}^{\dagger}, S_{I_{2}}^{\dagger}$ denote the collective atomic operators for the two ensembles $I_{1}$ and $I_{2}$ (or two metastable states in the same ensemble). The collective atomic excitations in the ensembles $I_{1}, L_{1}$ and $I_{2}, L_{2}$ are transferred to the optical excitations, which, after a $50 \%-50 \%$ beam splitter, are detected by the single-photon detectors $D_{1}^{I}, D_{1}^{L}$ and $D_{2}^{I}, D_{2}^{L}$. If there are a click in $D_{1}^{I}$ or $D_{1}^{L}$ and a click in $D_{2}^{I}$ or $D_{2}^{I}$, the protocol is successful. A $\pi$-phase rotation is then performed on the collective mode of the ensemble $R_{2}$ conditional on that the two clicks appear in the detectors $D_{1}^{I}, D_{2}^{L}$ or $D_{2}^{I}, D_{1}^{L}$. The collective excitation in the ensembles $R_{1}$ and $R_{2}$, if appearing, would be found in the same "polarization" state $\left(d_{0} S_{R_{1}}^{\dagger}+d_{1} S_{R_{2}}^{\dagger}\right)\left|0_{a} 0_{a}\right\rangle_{R_{1} R_{2}}$.

can also check that in Fig. 3, the phase shift $\psi_{\Lambda}(\Lambda=L$ or $R)$ together with the corresponding beam splitter operation are equivalent to a single-bit rotation in the basis $\left\{|0\rangle_{A} \equiv S_{\Lambda_{1}}^{\dagger}\left|0_{a} 0_{a}\right\rangle_{\Lambda_{1} A_{2}},|1\rangle_{A} \equiv S_{\Lambda_{2}}^{\dagger}\left|0_{a} 0_{a}\right\rangle_{\Lambda_{1} A_{2}}\right\}$ with the rotation angle $\theta=\psi_{\Lambda} / 2$. Now, it is clear how to do quantum cryptography and Bell inequality detection since we have the PME state and we can perform the desired single-bit 
rotations in the corresponding basis. For instance, to distribute a quantum key between the two remote sides, we simply choose $\psi_{\Lambda}$ randomly from the set $\{0, \pi / 2\}$ with an equal probability, and keep the measurement results (to be 0 if $D_{1}^{A}$ clicks, and 1 if $D_{1}^{A}$ clicks) on both sides as the shared secret key if the two sides become aware that they have chosen the same phase shift after the public declare. This is exactly the Ekert scheme [1] and its absolute security follows directly from the proofs in $[16,17]$. For the Bell inequality detection, we infer the correlations $E\left(\psi_{L}, \psi_{R}\right) \equiv P_{D_{1}^{L} D_{1}^{R}}+P_{D_{2}^{L} D_{2}^{R}}-P_{D_{1}^{L} D_{2}^{R}}-P_{D_{2}^{L} D_{1}^{R}}=\cos \left(\psi_{L}-\psi_{R}\right)$ from the measurement of the coincidences $P_{D_{1}^{L} D_{1}^{R}}$ etc. For the setup shown in Fig. 3a, we would have $|E(0, \pi / 4)+E(\pi / 2, \pi / 4)+E(\pi / 2,3 \pi / 4)-E(0,3 \pi / 4)|=2 \sqrt{2}$, whereas for any local hidden variable theories, the Clauser-Horn-Shimony-Holt (CHSH) inequality [18] implies that this value should be below 2 .

We can also use the established long-distance EME states for faithful transfer of unknown quantum states through quantum teleportation, with the setup shown by Fig. 3b. In this setup, if two detectors click on the left side, there is a significant probability that there is no collective excitation on the right side since the product of the EME states $\rho_{L_{1} R_{1}} \otimes \rho_{L_{2} R_{2}}$ contains vacuum components. However, if there is a collective excitation appearing from the right side, its "polarization" state would be exactly the same as the one input from the left. Therefore, as in the Innsbruck experiment [19], the teleportation here is probabilistic and needs posterior confirmation; but if it succeeds, the teleportation fidelity would be nearly perfect since in this case the entanglement is equivalently described by the PME state (4). The success probability for the teleportation is also given by $p_{a}=1 /\left[2\left(c_{n}+1\right)^{2}\right]$, which determines the average number of repetitions for a successful teleportation.

\section{Noise and built-in entanglement purification}

We next discuss noise and imperfections in the schemes for entanglement generation, connection, and applications. In particular, we show that each step contains built-in entanglement purification which makes the whole scheme resilient to the realistic noise and imperfections.

In the entanglement generation, the dominant noise is the photon loss, which includes the contributions from the channel attenuation, the spontaneous emissions in the atomic ensembles (which results in the population of the collective atomic mode $s$ with the accompanying photon going to other directions), the coupling inefficiency of the Stokes signal into and out of the channel, and the inefficiency of the single-photon detectors. The loss probability is denoted by $1-\eta_{p}$ with the overall efficiency $\eta_{p}=\eta_{p}^{\prime} \mathrm{e}^{-L_{0} / L_{\text {att }}}$, where we have separated the channel attenuation $\mathrm{e}^{-L_{0} / L_{\text {att }}}$ ( $L_{\text {att }}$ is the channel attenuation length) from other noise contributions $\eta_{p}^{\prime}$ with $\eta_{p}^{\prime}$ independent of the communication distance $L_{0}$. The photon loss decreases the success probably for getting a detector click from $p_{c}$ to $\eta_{p} p_{c}$, but it has no influence on the resulting EME state. Due to this noise, the entanglement 
preparation time should be replaced by $T_{0} \sim t_{\Delta} / \eta_{p} p_{c}$. The second source of noise comes from the dark counts of the single-photon detectors. The dark count gives a detector click, but without population of the collective atomic mode, so it contributes to the vacuum coefficient in the EME state. If the dark count comes up with a probability $p_{d c}$ for the time interval $t_{\Delta}$, the vacuum coefficient is given by $c_{0}=p_{d c} / \eta_{p} p_{c}$, which is typically much smaller than 1 since the Raman transition rate is much larger than the dark count rate. The final source of noise, which influences the fidelity to get the EME state, is caused by the event that more than one atom are excited to the collective mode $S$ whereas there is only one click in $D 1$ or $D 2$. The conditional probability for that event is given by $p_{c}$, so we can estimate the fidelity imperfection $\Delta F_{0} \equiv 1-F_{0}$ for the entanglement generation by

$$
\Delta F_{0} \sim p_{c} .
$$

Note that by decreasing the excitation probability $p_{c}$, one can make the fidelity imperfection closer and closer to zero with the price of a longer entanglement preparation time $T_{0}$. This is the basic idea of the entanglement purification. Therefore, in this scheme, the confirmation of the click from the single-photon detector generates and purifies entanglement at the same time.

In the entanglement swapping, the dominant noise is still the losses, which include the contributions from the detector inefficiency, the inefficiency of the excitation transfer from the collective atomic mode to the optical mode [20, 21], and the small decay of the atomic excitation during the storage [20, 21]. Note that by introducing the detector inefficiency, we have automatically taken into account the imperfection that the detectors cannot distinguish the single and the two photons. With all these losses, the overall efficiency in the entanglement swapping is denoted by $\eta_{s}$. The loss in the entanglement swapping gives contributions to the vacuum coefficient in the connected EME state, since in the presence of loss a single detector click might result from two collective excitations in the ensembles $I_{1}$ and $I_{2}$, and in this case, the collective modes in the ensembles $L$ and $R$ have to be in a vacuum state. After taking into account the realistic noise, we can specify the success probability and the new vacuum coefficient for the $i$-th entanglement connection by the recursion relations $p_{i} \equiv f_{1}\left(c_{i-1}\right)=\eta_{s}\left(1-\frac{\eta_{s}}{2\left(c_{i-1}+1\right)}\right) /\left(c_{i-1}+1\right)$ and $c_{i} \equiv f_{2}\left(c_{i-1}\right)=2 c_{i-1}+1-\eta_{s}$. The coefficient $c_{0}$ for the entanglement preparation is typically much smaller than $1-\eta_{s}$, then we have $c_{i} \approx\left(2^{i}-1\right)\left(1-\eta_{s}\right)=\left(L_{i} / L_{0}-1\right)\left(1-\eta_{s}\right)$, where $L_{i}$ denotes the communication distance after $i$ times entanglement connection. With the expression for the $c_{i}$, we can easily evaluate the probability $p_{i}$ and the communication time $T_{n}$ for establishing an EME state over the distance $L_{n}=2^{n} L_{0}$. After the entanglement connection, the fidelity of the EME state also decreases, and after $n$ times connection, the overall fidelity imperfection $\Delta F_{n} \sim 2^{n} \Delta F_{0} \sim\left(L_{n} / L_{0}\right) \Delta F_{0}$. We need fix $\Delta F_{n}$ to be small by decreasing the excitation probability $p_{c}$ in Eq. (5).

It is important to point out that our entanglement connection scheme also has built-in entanglement purification function. This can be understood as follows. 
Each time we connect entanglement, the imperfections of the setup decrease the entanglement fraction $1 /\left(c_{i}+1\right)$ in the EME state. However, the entanglement fraction decays only linearly with the distance (the number of segments), which is in contrast to the exponential decay of the entanglement for the connection schemes without entanglement purification. The reason for the slow decay is that in each time of the entanglement connection, we need to repeat the protocol until there is a detector click, and the confirmation of a click removes part of the added vacuum noise since larger vacuum components in the EME state result in more times of repetitions. The built-in entanglement purification in the connection scheme is essential for the polynomial scaling law of the communication efficiency.

As in the entanglement generation and connection schemes, our entanglement application schemes also have built-in entanglement purification which makes them resilient to the realistic noise. Firstly, we have seen that the vacuum components in the EME states are removed from the confirmation of the detector clicks and thus have no influence on the fidelity of all the application schemes. Secondly, if the single-photon detectors and the atom-to-light excitation transitions in the application schemes are imperfect with the overall efficiency denoted by $\eta_{a}$, one can easily check that these imperfections only influence the efficiency to get the detector clicks with the success probability replaced by $p_{a}=\eta_{a} /\left[2\left(c_{n}+1\right)^{2}\right]$, and have no effects on the communication fidelity. Finally, we have seen that the phase shifts in the stationary channels and the small asymmetry of the stationary setup are removed automatically when we project the EME state to the PME state, and thus have no influence on the communication fidelity.

The noise not correctable by our scheme includes the detector dark count in the entanglement connection and the non-stationary channel noise and set asymmetries. The resulting fidelity imperfection from the dark count increases linearly with the number of segments $L_{n} / L_{0}$, and form the non-stationary channel noise and set asymmetries increases by the random-walk law $\sqrt{L_{n} / L_{0}}$. For each time of entanglement connection, the dark count probability is about $10^{-5}$ if we make a typical choice that the collective emission rate is about $10 \mathrm{MHz}$ and the dark count rate is $10^{2} \mathrm{~Hz}$. Therefore, this noise is negligible even if we have communicated over a long distance ( $10^{3}$ the channel attenuation length $L_{\text {att }}$ for instance). The non-stationary channel noise and setup asymmetries can also be safely neglected for such a distance. For instance, it is relatively easy to control the non-stationary asymmetries in local laser operations to values below $10^{-4}$ with the use of accurate polarization techniques [22] for Zeeman sublevels (as in Fig. 2b).

\section{Scaling of the communication efficiency}

We have shown that each of our entanglement generation, connection, and application schemes has built-in entanglement purification, and as a result of this property, we can fix the communication fidelity to be nearly perfect, and at the 
same time keep the communication time to increase only polynomially with the distance. Let us assume that we want to communicate over a distance $L=L_{n}=2^{n} L_{0}$. By fixing the overall fidelity imperfection to be a desired small value $\Delta F_{n}$, the entanglement preparation time becomes $T_{0} \sim t_{\Delta} / \eta_{p} \Delta F_{0} \sim\left(L_{n} / L_{0}\right) t_{\Delta} / \eta_{p} \Delta F_{n}$. For an effective generation of the PME state (4), the total communication time $T_{\text {tot }} \sim T_{n} / p_{a}$ with $T_{n} \sim T_{0} \prod_{i=1}^{n}\left(1 / p_{i}\right)$. Therefore the total communication time scales with the distance by the law

$$
T_{\text {tot }} \sim \frac{2\left(L / L_{0}\right)^{2}}{\eta_{p} p_{a} \Delta F_{T} \prod_{i=1}^{n} p_{i}},
$$

where the success probabilities $p_{i}, p_{a}$ for the $i$-th entanglement connection and for the entanglement application have been specified before. The expression (6) has confirmed that the communication time $T_{\text {tot }}$ increases with the distance $L$ only polynomially. We show this explicitly by taking two limiting cases. In the first case, the inefficiency $1-\eta_{s}$ for the entanglement swapping is assumed to be negligibly small. One can deduce from Eq. (6) that in this case the communication time $T_{\text {tot }} \sim T_{\text {con }}\left(L / L_{0}\right)^{2} \mathrm{e}^{L_{\mathrm{o}} / L_{\text {att }}}$, with the constant $T_{\text {con }} \equiv 2 t_{\Delta} / \eta_{p}^{\prime} \eta_{a} \Delta F_{T}$ being independent of the segment and the total distances $L_{0}$ and $L$. The communication time $T_{\text {tot }}$ increases with $L$ quadratically. In the second case, we assume that the inefficiency $1-\eta_{s}$ is considerably large. The communication time in this case is approximated by $T_{\text {tot }} \sim T_{\text {con }}\left(L / L_{0}\right)^{\left[\log _{2}\left(L / L_{0}\right)+1\right] / 2+\log _{2}\left(1 / \eta_{s}-1\right)+2} \mathrm{e}^{L_{0} / L_{\text {att }}}$, which increases with $L$ still polynomially (or sub-exponentially in a more accurate language, but this makes no difference in practice since the factor $\log _{2}\left(L / L_{0}\right)$ is well bounded from above for any reasonably long distance). If $T_{\text {tot }}$ increases with $L / L_{0}$ by the $m$-th power law $\left(L / L_{0}\right)^{m}$, there is an optimal choice of the segment length to be $L_{0}=m L_{\text {att }}$ to minimize the time $T_{\text {tot }}$. As a simple estimation of the improvement in the communication efficiency, we assume that the total distance $L$ is about $100 L_{\text {att }}$, for a choice of the parameter $\eta_{s} \approx 2 / 3$, the communication time $T_{\text {tot }} / T_{\text {con }} \sim 10^{6}$ with the optimal segment length $L_{0} \sim 5.7 L_{\text {att }}$. This result is a dramatic improvement compared with the direct communication case, where the communication time $T_{\text {tot }}$ for getting a PME state increases with the distance $L$ by the exponential law $T_{\text {tot }} \sim T_{\text {con }} \mathrm{e}^{L / L_{\text {att }}}$. For the same distance $L \sim 100 L_{\text {att }}$, one needs $T_{\text {tot }} / T_{\text {con }} \sim 10^{43}$ for direct communication, which means that for this example the present scheme is $10^{37}$ times more efficient.

\section{Summary}

In summary, in this section we explained the recent atomic ensemble scheme for implementation of quantum repeaters and long-distance quantum communication. The proposed technique allows to generate and connect the entanglement and use it in quantum teleportation, cryptography, and tests of the Bell inequalities. All of the elements of the scheme are within the reach of current experimental technology, and have the important property of built-in entanglement purification 
which makes them resilient to the realistic noise. As a result, the overhead required to implement the scheme, such as the communication time, scales polynomially with the channel length. This is in remarkable contrast to direct communication where the exponential overhead is required. Such an efficient scaling, combined with a relative simplicity of the proposed experimental setup, opens up realistic prospective for quantum communication over long distances.

\section{References}

[1] A. Ekert, Phys. Rev. Lett. 67, 661 (1991).

[2] C.H. Bennett, G. Brassard, C. Crepeau, R. Jozsa, A. Peres, W.K. Wootters, Phys. Rev. Lett. 70, 1895 (1993).

[3] C.H. Bennett, G. Brassard, S. Popescu, B. Schumacher, J.A. Smolin, W.K. Wootters, Phys. Rev. Lett. 76, 722 (1996).

[4] H.J. Briegel, W. Dur, J.I. Cirac, P. Zoller, Phys. Rev. Lett. 81, 5932 (1998); W. Dur, H.J. Briegel, J.I. Cirac, P. Zoller, Phys. Rev. A 59, 169 (1999).

[5] E. Knill, R. Laflamme, W.H. Zurek, Science 279, 342 (1998).

[6] J. Preskill, Proc. R. Soc. Lond. A 454, 385 (1998).

[7] M. Zukowski, A. Zeilinger, M.A. Horne, A. Ekert, Phys. Rev. Lett. 71, 4287 (1993).

[8] J.I. Cirac, P. Zoller, H.J. Kimble, H. Mabuchi, Phys. Rev. Lett. 78, 3221 (1997).

[9] S.J. Enk, J.I. Cirac, P. Zoller, Science 279, 205 (1998).

[10] J. Ye, D.W. Vernooy, H.J. Kimble, Phys. Rev. Lett. 83, 4987 (1999).

[11] C.J. Hood, T.W. Lynn, A.C. Doherty, A.S. Parkins, H.J. Kimble, Science 287, $1447(2000)$.

[12] P.W.H. Pinkse, T. Fischer, T.P. Maunz, G. Rempe, Nature 404, 365 (2000).

[13] L.-M. Duan, M.D. Lukin, J.I. Cirac, P. Zoller, quant-ph/0105105, submitted to Nature.

[14] C. Cabrillo, J.I. Cirac, P. Garcia-Fernandez, P. Zoller, Phys. Rev. A 59, 1025 (1999).

[15] S. Bose, P.L. Knight, M.B. Plenio, V. Vedral, Phys. Rev. Lett. 83, 5158 (1999).

[16] H.K. Lo, H.F. Chau, Science 283, 2050 (1999).

[17] P.W. Shor, J. Preskill, Phys. Rev. Lett. 85, 441 (2000).

[18] J.F. Clauser, M.A. Horne, A. Shimony, R.A. Holt, Phys. Rev. Lett. 23, 880 (1969).

[19] D. Bouwmeester, J.-W. Pan, K. Mattle, M. Eibl, H. Weinfurter, A. Zeilinger, Nature 390, 575 (1997).

[20] C. Liu, Z. Dutton, C.H. Behroozi, L.V. Hau, Nature 409, 490 (2001).

[21] D.F. Phillips, A. Fleischhauer, A. Mair, R.L. Walsworth, M.D. Lukin, Phys. Rev. Lett. 86, 783 (2001).

[22] D. Budker, V. Yashuk, M. Zolotorev, Phys. Rev. Lett. 81, 5788 (1998). 